\title{
Co-Channel Coexistence Analysis between 5G IoT System and Fixed-Satellite Service at $40 \mathrm{GHz}$
}

\author{
Xi Meng $\mathbb{D}^{1},{ }^{1}$ Liyuan Zhong $\left(\mathbb{D},{ }^{2}\right.$ Dong Zhou $\mathbb{D}^{2},{ }^{2}$ and Dacheng Yang ${ }^{1}$ \\ ${ }^{1}$ School of Information and Communication Engineering, Beijing University of Posts and Telecommunications, \\ Beijing 100876, China \\ ${ }^{2}$ Wireless Product Operation Division in ZTE Corporation, Xi'an 710114, China \\ Correspondence should be addressed to Xi Meng; ximeng@bupt.edu.cn
}

Received 3 April 2019; Accepted 10 September 2019; Published 7 October 2019

Guest Editor: Bo Rong

Copyright (c) 2019 Xi Meng et al. This is an open access article distributed under the Creative Commons Attribution License, which permits unrestricted use, distribution, and reproduction in any medium, provided the original work is properly cited.

\begin{abstract}
It is a promising way to successfully operate the fifth generation $(5 \mathrm{G})$ system with Internet of Things (IoT) in potential mmWave spectrum bands. This paper investigates the intelligent co-channel coexistence between the 5G IoT system and the fixed-satellite service (FSS) system at $40 \mathrm{GHz}$. The key issue, as identified, is the accurate estimation of interference based on mmWave propagation characteristics. Our simulation results reveal that interference from the 5G IoT system into the FSS ground stations can be kept below the protection threshold by considering different deployment parameters, such as antenna patterns, height of Earth station (ES), and separation distance.
\end{abstract}

\section{Introduction}

In the past, millimeter wave (mmWave) technologies were widely researched and deemed suitable for applications in space services, especially for different kinds of satellite communication services such as fixed-satellites service (FSS), mobile-satellite service (MSS), and inter-satellite service (ISS) [1]. However, today, the growth demand for both a multi-gigabits-per-second (multi-Gbps) rate and the involvement of IoT terminals becomes a major challenge for next-generation International Mobile Telecommunications (IMT) networks $(5 G)[2,3]$. In order to satisfy these requirements, it is necessary to introduce the mmWave, such as that at $40 \mathrm{GHz}$, to the $5 \mathrm{G} \mathrm{IoT}$ system, as these bands provide an ultrawide band spectrum, the potential for spatial densification, and the ability to access a massive number of IoT terminals $[4,5]$.

One of the main difficulties in deploying mmWave technology is the protection of incumbents. Thus, it is essential to have an intelligent coexistence solution between the 5G IoT system and the FSS system in the same geographical area at $40 \mathrm{GHz}$, based on the exploration of the interference. Our study considers only the downlink scenario, as ITU-R allocates other bands for FSS uplink transmission [6]. In particular, the interference into an ES receiver antenna may be generated by each single wireless transmission from base stations (BSs) to their associated IoT terminals and from the IoT terminals to their BSs.

The key issue for intelligent co-channel coexistence, as identified, is the accurate estimation of interference based on mmWave propagation characteristics. Interference estimation determines whether the $5 \mathrm{G}$ IoT system can share the same band with a predeployed FSS system. For example, if studies have shown that the two systems will generate unavoidable interference with each other, then RF engineers must design additional functionalities to mitigate or suppress this sort of interference. In contrast, if the spectrum sharing study concludes that both 5G IoT and FSS systems can coexist without generating any interference with each other, then it will be unnecessary to introduce interference mitigating technologies with extra overhead.

Initial results can be achieved based on previous preliminary coexistence analyses between the 5G system and existing services [7-15]. This paper, however, varies from previous studies in many ways. First, the results obtained by some previous researchers [7-9] focused on the relationship 
between the advanced IMT system, also called the $4 \mathrm{G}$ system, and FSS; the target frequency was mainly from a $3.5 \mathrm{GHz}$, non-mmWave band, which is obviously different from the current research goals. Second, unlike the study undertaken in the current work, other previous research [10-13] has primarily considered the $5 \mathrm{G}$ scenario to be a traditional enhanced mobile broadband (eMBB) scenario rather than a 5G IoT scenario, such as ultrareliable and low latency (URLLC) and massive machine-type communications (mMTC). Finally, the findings by some scholars $[14,15]$ were obtained only from theoretical and deterministic analyses, whereas this paper additionally provides a simulation analysis and related evaluation methodology.

The reminder of this paper is organized as follows. Section 2 investigates the typical coexistence scenario between the 5G IoT system and the FSS system. Section 3 presents the simulation results by comparing a variety of propagation models and related methodologies. Finally, Section 4 concludes the paper with future research directions.

\section{System Model and Coexistence Scenarios}

2.1. Typical Coexistence Scenario. The coexistence scenario and related network topology are introduced to provide a direct view into the coexistence issue of the $5 \mathrm{G}$ IoT system and the FSS system.

Figure 1 illustrates the coexistence scenario for the $5 \mathrm{G}$ IoT system and the FSS system. More specifically, the 5G IoT system shares frequency bands from 37.5 to $42.5 \mathrm{GHz}$ with the FSS system downlink. Thus, it is very likely that the FSS ES will be interfered with by the signals of the BSs and terminals of the $5 \mathrm{G}$ IoT system, which is reflected by the red dashed arrows. Moreover, the red grid indicates the separation distance between the $5 \mathrm{G}$ IoT system and the FSS system, the definition of which will be discussed in the next section of the paper. Because of the limited transmitting power of satellites, indicated by the black line, and the extremely long transmission distance between the satellite and the ES, the interference of the receiving FSS downlink signal will be ignored by the $5 \mathrm{G}$ IoT system because the signal is too weak to cause interference to it. In addition, the potential interference resource from the FSS uplink signal can mostly be ignored due to the obvious frequency isolation between the FSS uplink and 5G IoT system. It is important to note that only interfered signals from BSs are evaluated in this paper. The main reason for this is that the transmitted power of IoT terminals is much lower than that of base stations.

Based on analysis of the coexistence scenario, related topology should be considered for further evaluation. A typical network topology is presented in Figure 2, and it corresponds to the downlink band of the FSS system at $40 \mathrm{GHz}$. The evaluated area is close to a circle with a surface area of approximately $1000 \mathrm{~km}^{2}$, which represents the developed area of a large city. The inner part of the city is an urban region with a radius of $8 \mathrm{~km}$, and the outer part of the city is a suburban region with a radius of $18 \mathrm{~km}$. It is notable that at least one BS is located on the ring with a radius of

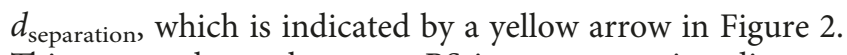
This means that at least one BS is at a separation distance from the FSS Earth station, because this type of topology is the worst case for the coexistence analysis between the $5 \mathrm{G}$ IoT and FSS systems.

Moreover, the FSS ES is placed at the center of the circle. Additionally, it should be noted that $40 \mathrm{GHz}$, as a millimeter wave, will mostly be deployed at the hotspot rather than exhibiting seamless coverage throughout the $5 \mathrm{G}$ IoT network; therefore, the number of BSs is wholly related to the ratio of hotspot areas to the area of a city. The total BS number $\left(N_{\text {IMT }}\right)$ in the simulation is derived from the following equation:

$$
N_{\mathrm{IMT}}=S_{\mathrm{urb}} *\left(D_{s_{\mathrm{urb}}} * R_{a_{\mathrm{urb}}}\right)+S_{\mathrm{sub}} *\left(D_{s_{\mathrm{urb}}} * R_{a_{\mathrm{urb}}}\right)
$$

where $N_{\text {IMT }}$ expresses the total number of IMT BSs in the simulation, $S_{\text {urb }}$ and $S_{\text {sub }}$ refer to the sizes of the urban and suburban areas in $\mathrm{km}^{2}$, respectively, and $D_{s_{\text {urb }}}$ and $D_{s_{\text {sub }}}$ are the density values for the outdoor urban and suburban hotspot areas, respectively, i.e., the density of simultaneously transmitting the IoT terminal or number of BSs per $\mathrm{km}^{2}$. Finally, $R_{a_{\text {urb }}}$ and $R_{a_{\text {sub }}}$ represent the ratios of the hotspot areas to the urban and suburban areas, respectively. In Table 1, one set of typical values for equation (1) is presented, and these values are also considered for coexistence analysis in next chapter.

2.2. Model of the 5G IoT System with Massive MIMO. The 5G IoT system will use frequency bands both below and above $6 \mathrm{GHz}$. However, higher frequency bands, especially that at $40 \mathrm{GHz}$, are suitable for the IoT system in 5G, as a wider bandwidth which supports access to massive number of IoT terminals. Moreover, mmWave better meets the demands of different kinds of IoT devices by beamforming, which is one of the key technologies in $5 \mathrm{G}$.

The 5G IoT system with beamforming is a massive MIMO wireless system [16] that employs a combination of limited feedback as illustrated in Figure 3. $N_{t}$ transmitting antennas and $N_{r}$ receiving antennas are used at transmitter and receiver, respectively. Figure 3 shows the antenna configuration of the $5 \mathrm{G}$ IoT system, where $V$-Ant. denotes the antenna with vertical $(V)$ polarization, and $H$-Ant. denotes the antenna with horizontal $(H)$ polarization. The wireless channel matrix is described by an input-output relation with $H$ to $H, H$ to $V, V$ to $V$, and $V$ to $H$ polarized waves, based on the flat fading assumption.

More specifically, the signal $y$ received by the IoT terminal from the $5 \mathrm{G}$ BS is represented as follows:

$$
y=\sqrt{\rho} \mathbf{H} f x+n,
$$

where $f$ is the precoding matrix at the transmitter side, $n$ is the $N_{\mathrm{r}}$-dimensional noise vector, which has independent and identically distributed entries with normal distribution $\mathrm{CN}$ $(0,1), s$ represents the transmitted symbol with normalized energy, and $\rho$ is the signal-to-noise ratio (SNR). The wireless channel $\mathbf{H}=H(t)$ is a matrix that describes the dual-polarized MIMO channel. Its modelling method is discussed in 


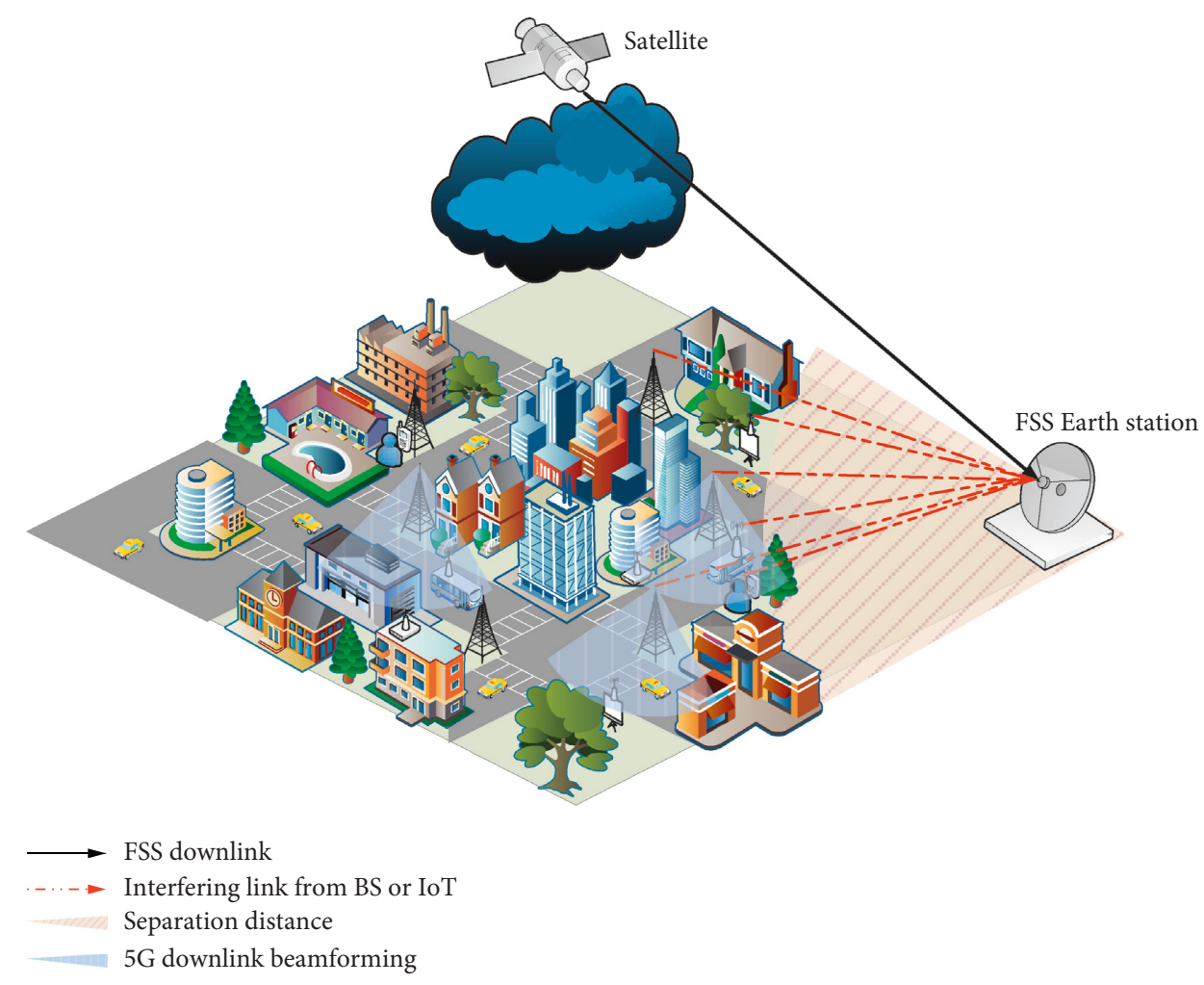

FIGURE 1: Illustration of 5G IoT sharing scenario with FSS.
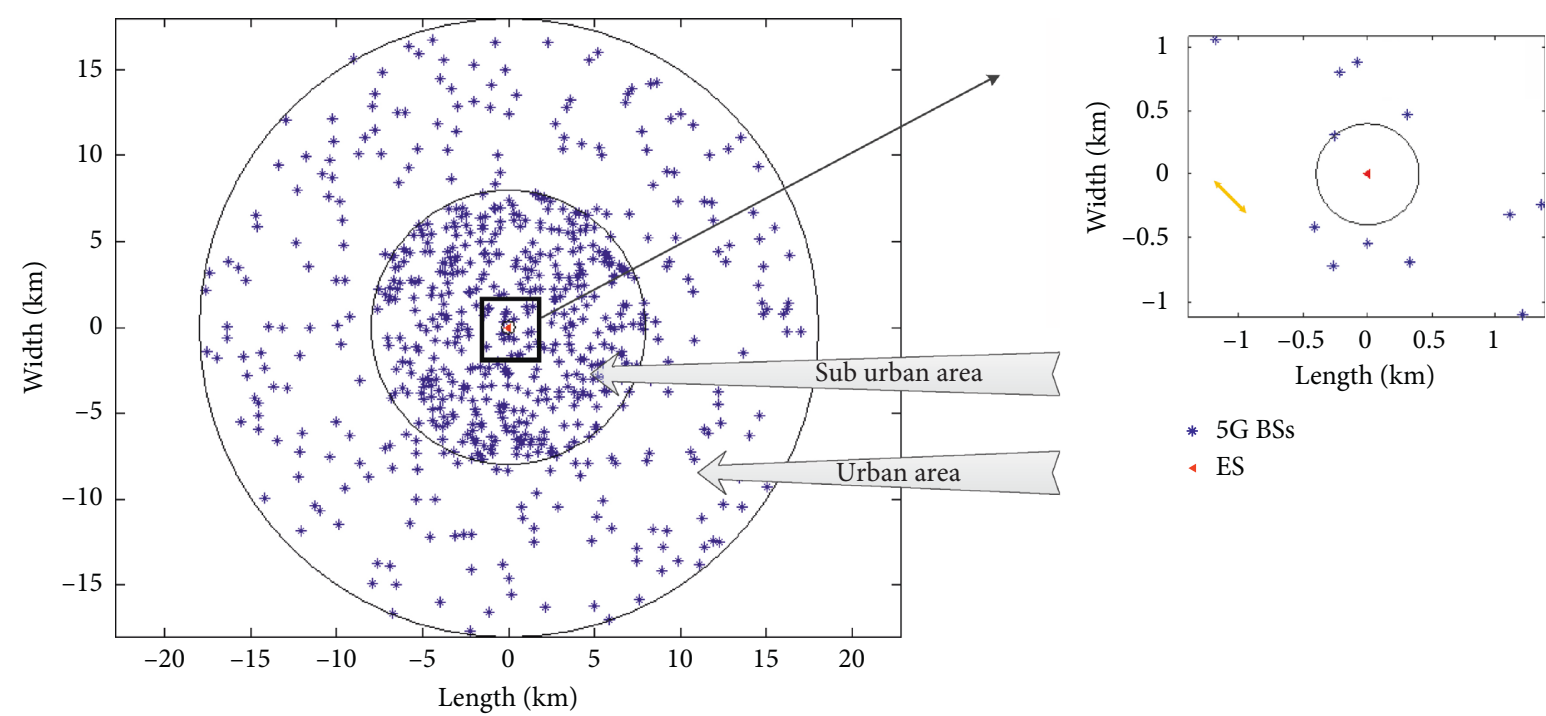

* 5G BSs

$4 \mathrm{ES}$

* 5G BSs

$4 \mathrm{ES}$

FIgUre 2: Topology of 5G IoT and FSS ES coexistence analysis.

Table 1: Typical value of $D_{s}$ and $R_{a}$.

\begin{tabular}{lcr}
\hline & Outdoor urban hotspot & Outdoor suburban hotspot \\
\hline Density (devices per $\left.\mathrm{km}^{2}\right)$ & $D_{s_{\text {urb }}}=30$ & $D_{s_{\text {sub }}}=10$ \\
Ratio (\%) & $R_{a_{\text {urb }}}=7$ & $R_{a_{\text {sub }}}=3$ \\
\hline
\end{tabular}




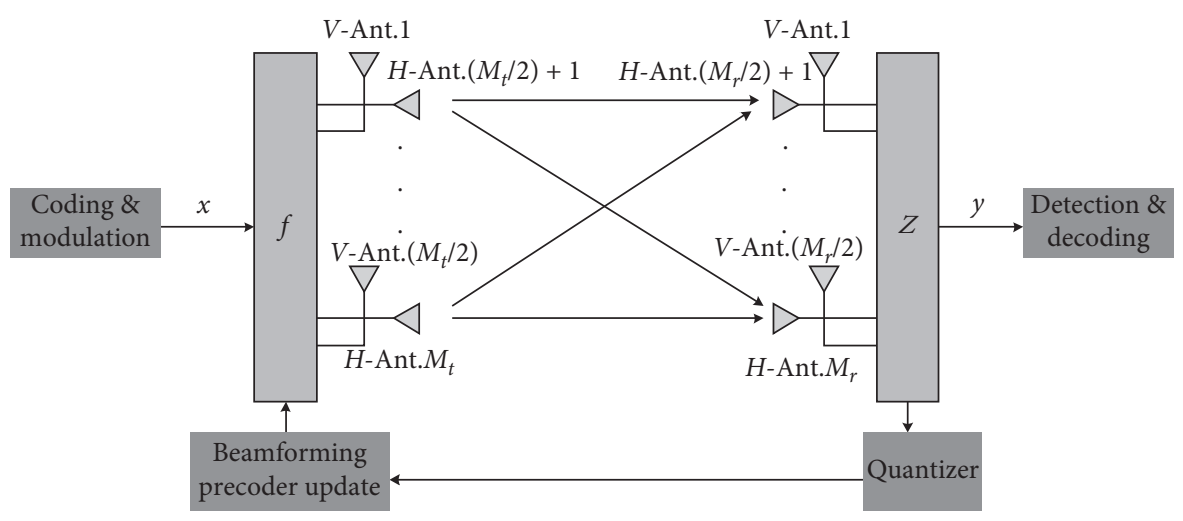

Figure 3: 5G IoT system model.

previous reports $[17,18]$, and any two receiving antenna channels $(u, x)$ between the responses can be formulated as

$$
\begin{aligned}
& H(t)=\sqrt{P_{n}} \sum_{m=1}^{M}\left[\begin{array}{l}
F_{r x, u, V}\left(\varphi_{n, m}, \gamma_{n, m}\right) \\
F_{r x, u, H}\left(\varphi_{n, m}, \gamma_{n, m}\right)
\end{array}\right]^{\mathrm{T}} \\
& \cdot\left[\begin{array}{cc}
\exp \left(j \Phi_{n, m}^{v v}\right) & \sqrt{\kappa} \exp \left(j \Phi_{n, m}^{v h}\right) \\
\sqrt{\kappa} \exp \left(j \Phi_{n, m}^{h v}\right) & \exp \left(j \Phi_{n, m}^{h h}\right)
\end{array}\right] \\
& \cdot\left[\begin{array}{l}
F_{t x, u, V}\left(\phi_{n, m}, \theta_{n, m}\right) \\
F_{t x, u, H}\left(\phi_{n, m}, \theta_{n, m}\right)
\end{array}\right] \\
& \cdot \exp \left(j 2 \pi \lambda_{0}^{-1} \bar{r}_{s} \cdot \bar{\Phi}_{n, m}\right) \exp \left(j 2 \pi \lambda_{0}^{-1} \bar{r}_{u} \cdot \bar{\Psi}_{n, m}\right) \\
& \cdot \exp \left(j k\|v\| \cos \left(\theta_{n, m, \mathrm{AoA}}-\theta_{v}\right) t\right) \text {, }
\end{aligned}
$$

where both $n$ and $m$ refer to the index of the cluster and each ray and $P_{n}$ is the ray power in the $n^{\text {th }}$ cluster. Additionally, $\varphi_{n, m}, \gamma_{n, m}$ represents the angles of arrival of the $m^{\text {th }}$ ray, whereas $\phi_{n, m}, \theta_{n, m}$ expresses the angles of divergence of the $m^{\text {th }}$ ray in the $n^{\text {th }}$ cluster. The $F_{r x, u, V}$ and $F_{r x, u, H}$ functions are the multipath directions of $\varphi_{n, m}, \gamma_{n, m}$. Corresponding to the vertical and horizontal polarization antenna gain, $\kappa$ represents the cross polarization power ratio in linear scale and $\lambda_{0}^{-1}$ expresses the carrier frequency. Moreover, $\exp \left(j \Phi_{n, m}^{v v}\right)$ and $\exp \left(j \Phi_{n, m}^{h h}\right)$, respectively, represent the phase response of each ray in the randomly generated vertical polarization direction and horizontal plane, whereas $\exp \left(j \Phi_{n, m}^{v h}\right)$ and $\exp \left(j \Phi_{n, m}^{h v}\right)$ represent each horizontal to vertical, and vertical to horizontal polarization component leaked corresponding to random phase response. Then, $\bar{r}_{u}$ and $\bar{r}_{s}$ express the receiving end and the end of the antenna sends. $\bar{\Phi}_{n, m}$ and $\bar{\Psi}_{n, m}$ are vector expressions for the angles of arrival and departure, respectively. Finally, the IoT terminal movement speed is expressed as $v$.

In this paper, the antenna array configuration of the BS was assumed to be 8 and 16 elements placed along the horizontal and vertical directions, respectively, and a set of the main BS parameters is presented in Table 2. More importantly, the composite antenna gain is important for coexistence analysis [17] because it is the resulting beamforming antenna pattern from the logarithmic sum of the array gain in massive MIMO in mmWave. For example, the composite antenna gain can be formulated as

$$
G_{A}(\theta, \varphi)=G_{E}(\theta, \varphi)+10 \log \left(\left|\sum_{i=1}^{N_{H}} \sum_{j=1}^{N_{V}} w_{i, j} \cdot v_{i, j}\right|^{2}\right)
$$

where $G_{E}(\theta)$, represents the antenna element gain, $w_{i, j}$ is the weighting, and $v_{i, j}$ is the super position vector.

Based on equations (3) and (4), the antenna gain pattern for $126(8 \times 16)$ elements in the $5 \mathrm{G}$ IoT BS is shown in Figure 4. More specifically, its composite antenna gain pattern is three-dimensionally depicted, with the beam range covered from 0 to 180 degrees in vertical direction, but from -180 to 180 degrees in horizontal direction.

On the other hand, 4 antenna elements in both directions are assumed for the antenna array configuration of the IoT terminal. Additional related significant parameters of the IoT terminal can be found in Table 3, which also includes some important parameters of the BS. Furthermore, the antenna gain pattern was also created with the same method as that for the $5 \mathrm{G}$ BS and is demonstrated in Figure 5 . The composite antenna gain pattern of the IoT terminal with $16(4 \times 4)$ antenna elements is threedimensionally drawn too.

It is obvious from both Figures 4 and 5 that in the massive MIMO 5G IoT system, the width of the beam is narrower with the increase in the of number of antenna elements. The main reason for this is due to one of the advantage of massive MIMO in mmWave; although the coverage of the beam is limited, the transmitted power is more concentrated, which effectively improves the channel capacity. In other words, regarding sharing with the FSS ES system, the probability of interference will decrease, as it is difficult to point to the antenna of the ES from a narrower beam of the $5 \mathrm{G}$ IoT system.

2.3. Model of FSS System. FSS is one of the main applications in satellite communication. FSS provides the dual-transmission path between the satellite and its ES to provide telecommunication services including data, video, and voice transmission. In this paper, FSS-receiving ESs operate in the space-to-Earth direction from $40 \mathrm{GHz}$, more specifically from the 37.5 to $42.5 \mathrm{GHz}$ frequency bands. The main downlink ES parameters are presented in Table 4. 
TABLE 2: Key parameters for BS in 5G IoT system.

Parameter

Network topology and characteristics

Frequency reuse

Antenna height (radiation center)

Sectorization

Antenna down tilt

Antenna deployment

Network loading factor

BS TDD activity factor

Element gain $G_{E}(\theta, \varphi)$

$3 \mathrm{~dB}$ beam width of single element

Front-to-back ratio

Antenna polarization

Radiating element spacing

Array ohmic loss

Conducted power per antenna element

BS maximum coverage angle in the horizontal plane
Outdoor suburban hotspot/outdoor urban hotspot

$$
\text { Suburban: } 10 \mathrm{BS} / \mathrm{km}^{2}
$$$$
\text { Urban: } 30 \mathrm{BSs} / \mathrm{km}^{2}
$$

$$
6 \mathrm{~m}
$$

Single sector

10 degrees

Below rooftop $20 \%$

$80 \%$

$5 \mathrm{dBi}$

65 degrees for both $H / V$

$30 \mathrm{~dB}$ for both $H / V$

Linear $\pm 45^{\circ}$

0.5 of wavelength for both $H / V$

$$
3 \mathrm{~dB}
$$

$8 \mathrm{~dB}(\mathrm{~m} / 200 \mathrm{MHz})$

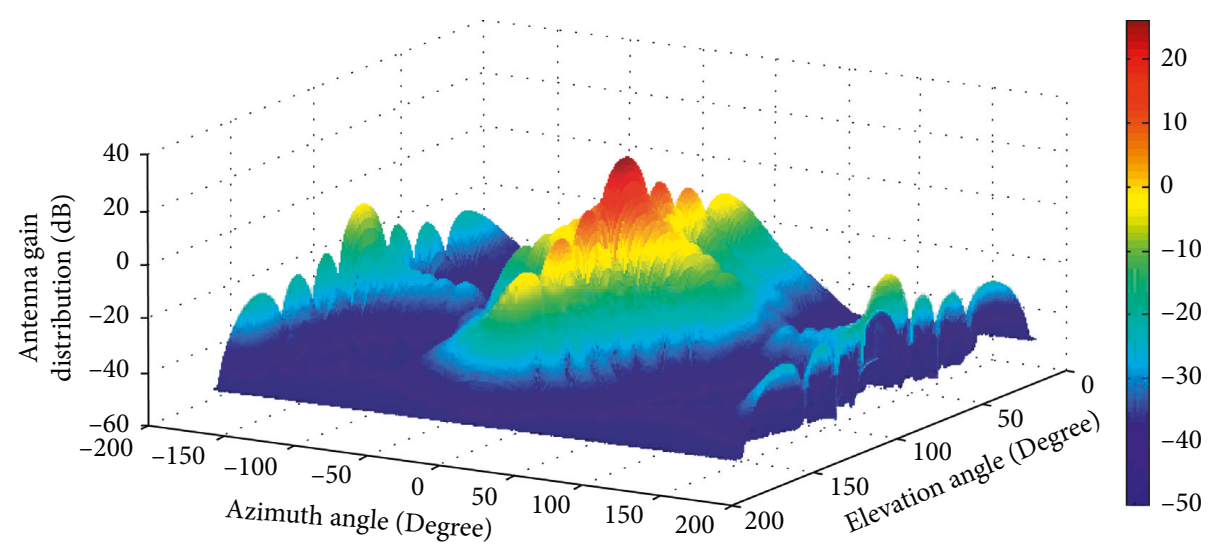

FIgURe 4: Composite antenna gain pattern for $5 \mathrm{G}$ BS with $8 \times 16$ antenna elements.

TABLE 3: Key parameters for terminal in the 5G IoT system.

Parameters

Network topology and characteristics

Body loss

Indoor IoT terminal usage

Terminals TDD activity factor

$3 \mathrm{~dB}$ beam width of single element

Front-to-back ratio

Antenna array configuration (row $\times$ column)

Maximum IoT terminal output power

Transmit power target value per $180 \mathrm{kHz}$

Path loss compensation factor $\alpha$

Furthermore, in order to conduct a sharing study between the 5G IoT system and the FSS system, it is assumed that the antenna elevation angle of the ES is chosen as the minimum value, i.e., 10 degrees. Although this value is pessimistic, it means fewer dropping locations of the ES's antenna pointing towards the satellite and always indicates the worst-case assumption in further coexistence analysis.
Outdoor suburban hotspot/outdoor urban hotspot Suburban: 30 terminals $/ \mathrm{km}^{2}$

Urban: 100 terminals $/ \mathrm{km}^{2}$

$$
\begin{gathered}
4 \mathrm{~dB} \\
5 \% \\
20 \% \\
90^{\circ} \text { for both } H / V \\
25 \mathrm{~dB} \text { for both } H / V \\
4 \times 4 \text { elements } \\
22 \mathrm{dBm} \\
-95 \mathrm{dBm} \\
1
\end{gathered}
$$

The FSS ES antenna gain is calculated as a function of the off-boresight angles [5, 18]. Assuming $\vartheta$ is the azimuth of the $5 \mathrm{G}$ BS or IoT terminal without the ES main lobe, the offboresight angles $\phi$ of the ES towards the BS or terminal can be computed as follows:

$$
\phi=\arccos (\cos (\alpha) \cos (\varepsilon) \cos (\vartheta)+\sin (\alpha) \sin (\varepsilon)),
$$




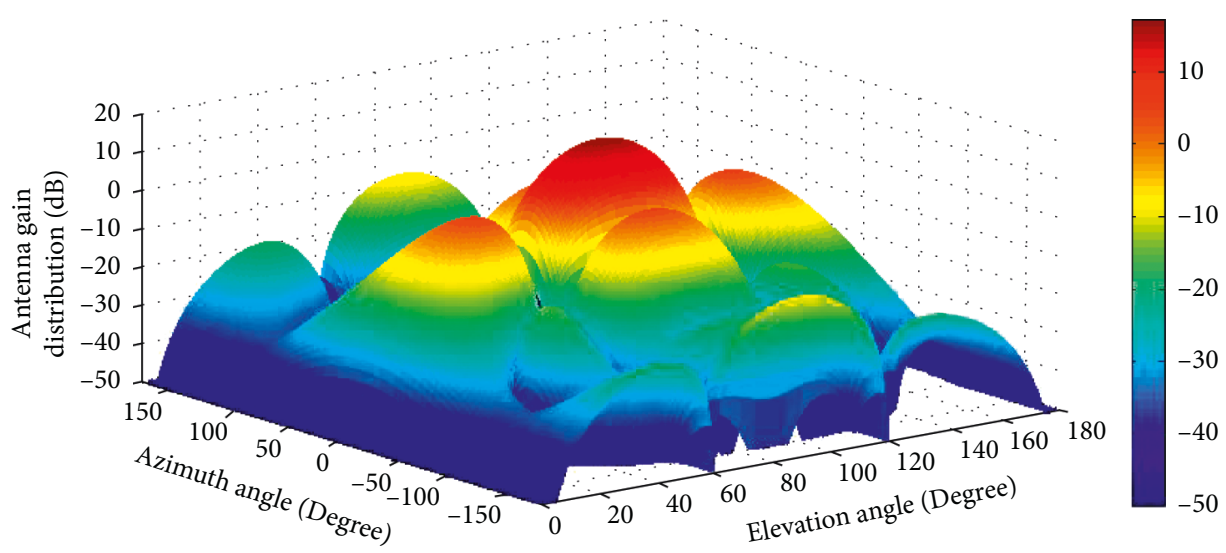

FIGURE 5: Composite antenna gain pattern for 5G IoT terminal with $4 \times 4$ antenna elements.

TABLE 4: Parameters for ES in FSS.

\begin{tabular}{lc}
\hline Parameter & Value \\
\hline Frequency range $(\mathrm{GHz})$ & $37.5-42.5$ \\
Noise bandwidth $(\mathrm{MHz})$ & $100-600$ \\
ES antenna diameter $(\mathrm{m})$ & 6.8 \\
Peak receive antenna gain $(\mathrm{dBi})$ & 68 \\
System receive noise temperature $(\mathrm{K})$ & 250 \\
Minimum ES elevation angle $($ degrees $)$ & 10 \\
Interference-to-noise ratio $I / N(\mathrm{~dB})$ & -12.2 \\
\hline
\end{tabular}

where $\alpha$ refers to the elevation angle. Additionally, $\epsilon$ is calculated as

$$
\varepsilon=\frac{h_{\mathrm{ES}}-h}{d}-\frac{d}{2 r}
$$

where $h$ and $h_{\mathrm{ES}}$ are defined as the heights of the 5G BS or IoT terminals and ES in meters, respectively, and $r$ is the effective Earth radius, approximately $8.5 \times 10^{3} \mathrm{~km}$. Therefore, the FSS ES off-boresight antenna gain pattern, the unit of which is $\mathrm{dBi}$, is calculated as

$$
G= \begin{cases}32-25 \log \varphi, & \text { for } 1^{\circ} \leq \varphi \leq 48^{\circ}, \\ -10, & \text { for } 48^{\circ} \leq \varphi \leq 360^{\circ} .\end{cases}
$$

\section{Coexistence Analysis Results for Intelligent Spectrum Sharing between 5G IoT System and FSS}

3.1. Propagation Model. The propagation model refers to the propagation fading effect, which is a significant model for studies of sharing between the 5G IoT and FSS systems. In this paper, the propagation model describes a ground-toground wireless communications system. More importantly, the model is designed as a prediction method for the evaluation of the interference between BSs or IoT terminals and ESs on the Earth surface at frequencies from about $0.1 \mathrm{GHz}$ to $50 \mathrm{GHz}$. The model should consider a set of propagation mechanisms, including attenuation by atmospheric gases, diffraction, free-space propagation, tropospheric scatter, clutter loss, and ducting reflection. However, since the propagation model assumes known information of the location of BSs, IoT terminals, and ES, its clutter loss model is inappropriate for our analysis. Therefore, an additional method to calculate cluster loss is presented below.

The clutter loss not exceeded for $\mathrm{p} \%$ of locations for the terrestrial to the terrestrial path [9], $L_{\mathrm{ctt}}(\mathrm{dB})$, is given by

$$
\begin{aligned}
L_{\mathrm{ctt}}= & -5 \log \left(10^{-0.2(23.5+9.6 \log (f))}\right. \\
& \left.+10^{-0.2(32.98+23.9 \log (d)+3 \log (f))}\right) \\
& -6 Q^{-1}\left(\frac{p}{100}\right),
\end{aligned}
$$

where $d$ is the total path length in $\mathrm{km}, f$ is the frequency in $\mathrm{GHz}$, and $Q^{-1}(p / 100)$ is the inverse complementary normal distribution function with $p$.

Based on the above introduced propagation mode, Figure 6 illustrates cluster loss in different distances and frequencies, in which the green line represents a cluster loss around $40 \mathrm{GHz}$; it is also applied in our simulation.

3.2. Methodology. In this section, a novel Monte Carlo evaluation methodology is introduced to analyze the aggregate interference from the $5 \mathrm{G}$ IoT system, including BSs and terminals, to a FSS ES.

First, the ES is located in the center, and random distributions of both BSs and the IoT terminal are generated according to the sharing study scenario mentioned previously. In addition, their interference links from the $5 \mathrm{G}$ BSs or IoT terminals to the ES also are produced. It is worth noting that the BSs and terminal's beams face toward each other due to the application beamforming and the randomness of the interference resource.

Second, the related parameters and antenna pattern, such as the transmit power and network load, should be configured and are presented in Tables 2-4 for both the $5 \mathrm{G}$ IoT and FSS systems.

Third, the interference model from the $i^{\text {th }} 5 \mathrm{G}$ BS or the IoT terminals linking to the FSS ES are shown by using the following equation:

$$
I_{\mathrm{IMT} \longrightarrow \mathrm{FSS}}\left(\theta_{t x}^{i}, \theta_{r x}^{i}\right)=\mathrm{PD}_{t x}-\mathrm{OL}_{t x}+G_{t x}\left(\theta_{t x}^{i}\right)-\mathrm{PL}+G_{r x}\left(\theta_{r x}^{i}\right),
$$




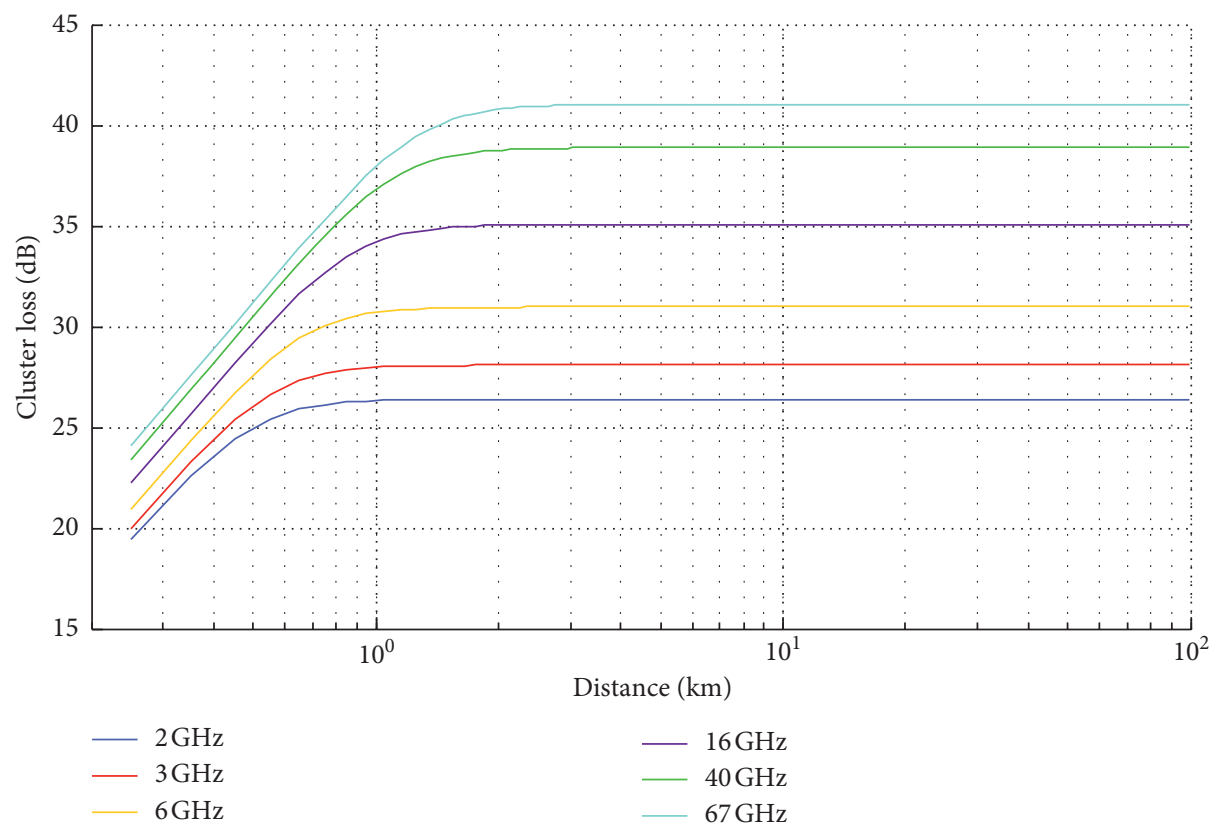

FIgURE 6: Cluster loss model with varying frequencies and distances for ES.

where $\mathrm{PD}_{t x}$ is the transmit station signal power density in $\mathrm{dBm} / \mathrm{MHz}, \mathrm{OL}_{t x}$ represents the transmit station array ohmic loss in $\mathrm{dB}$, and $G_{t x}\left(\theta_{t x}^{i}\right)$ is the $5 \mathrm{G}$ BS or IoT terminals' antenna gain in the direction of the ES, accounting for the beamforming antenna pattern in $\mathrm{dBi} . G_{r x}\left(\theta_{r x}^{i}\right)$ represents the antenna gain of the ES in the direction of the transmit station in $\mathrm{dBi}$, and $\mathrm{PL}$ refers to propagation loss, which includes path loss, clutter loss, and polarization discrimination in $\mathrm{dB}$.

Furthermore, the aggregate interference from all 5G BSs or IoT terminals at the FSS ES receiver level is calculated by the sum of the FSS ES from each interference link from equation (9).

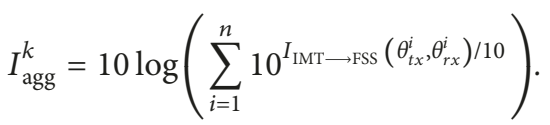

In equation (10), $I_{\text {agg }}^{k}$ represents the $k^{\text {th }}$ running simulation to obtain the aggregate external system interference towards the interfered-with system from all $n$ interference links in $\mathrm{dB}$.

Then, when $(m)$ numbers of simulations are run, the average aggregate interference, $I_{\text {ave }}^{m}$, can be calculated by the following equation:

$$
I_{\mathrm{ave}}^{m}=1-\log \left(\frac{\sum_{k=1}^{m} 10^{I_{\mathrm{agg}}^{k} / 10}}{m}\right) .
$$

Finally, the interference margin between the 5G IoT system and the FSS system can be calculated as

$$
D_{\text {margin }}=\left(\left(\frac{I}{N_{\mathrm{thr}}}\right)+N\right)-I_{\text {ave }}^{m},
$$

where $D_{\text {margin }}$ is the interference margin required by the $5 \mathrm{G}$ BS or IoT terminal, $I / N_{\text {thr }}$ is a criterion of the interference- noise ratio, which is presented in Table 5 , and $N$ is the thermal noise of the FSS ES. It is a common view that $\left(\left(I / N_{\text {thr }}\right)+N\right)$ is also called an interference threshold, $I_{\mathrm{thr}}$, in $\mathrm{dBm} / \mathrm{MHz}$.

3.3. Simulation Results. Figure 7 demonstrates the cumulative distribution functions (CDFs) of the antenna gain from the $5 \mathrm{G}$ IoT system towards the FSS ES, which describes the parameter $G_{t x}\left(\theta_{t x}^{i}\right)$ in equation (9). It is one of the most important factors that influence the final interference margin between the $5 \mathrm{G}$ IoT and FSS systems. More specifically, Figure 7(a) shows the CDF of the transmitted antenna gain from the BS, and Figure 7(b) displays the characteristics of the 5G IoT terminals' antenna gain in the direction of the receiver station while taking into account the beamforming antenna pattern by CDF. Moreover, from Figure $7(\mathrm{a})$, it is evident that the BS antenna gains are primarily distributed from approximately -75 to $25 \mathrm{~dB}$, and a flat, increasing curve can be seen at around the $95 \%$ point of the CDF. In contrast, the distribution of the antenna gain of the IoT terminal mainly decreases from about -70 to $15 \mathrm{~dB}$, but the trend at about the $95 \%$ point of the CDF increases more sharply than that of the BS. This indicates that it is possible to produce a higher aggregated gain with BSs because of the fewer number of antennas in the IoT terminals' antenna pattern.

Both Tables 6 and 7 demonstrate the results of the interference margin on links from different antenna patterns of a $5 \mathrm{G}$ BS to the ES. Table 8 presents the interference margin required by the IoT terminal. These results depend on different heights of the ES, isolation distances, and criteria of interference-noise ratios. The simulation details should be clarified. First, Monte Carlo simulations with 5000 iterations $(m=5000)$ can be considered due to the randomness of both the BS and IoT terminal positions in each sector. More importantly, these configurations of the BS and IoT terminals can be reused from Tables 6 and 7, respectively. In both these tables, varying 
TABLE 5: Interference criteria for sharing study between 5G IoT and FSS system in $40 \mathrm{GHz}$.

\begin{tabular}{lcc}
\hline Frequency ranges & $\begin{array}{c}\text { Percentage of time for which the } I / N_{\text {thr }} \text { value could } \\
\text { be exceeded }(\%)\end{array}$ & $I / N_{\text {thr }}$ criteria $(\mathrm{dB})$ \\
\hline $37.5-51.4 \mathrm{GHz}$ & 20 & -10 \\
\hline
\end{tabular}

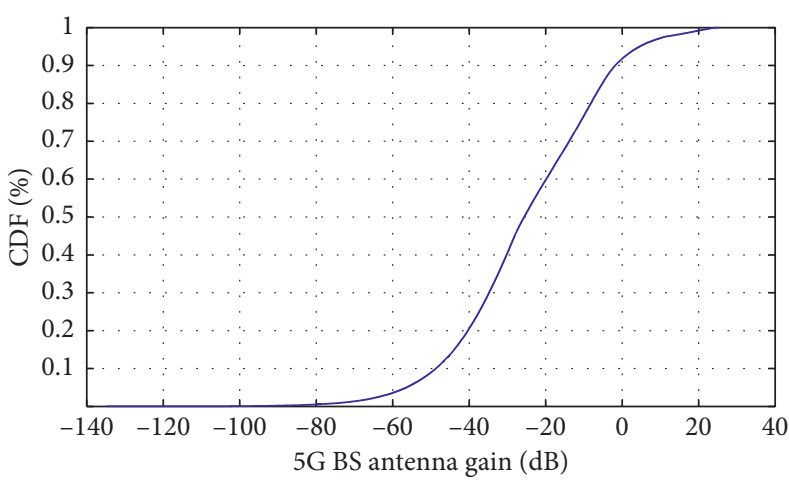

(a)

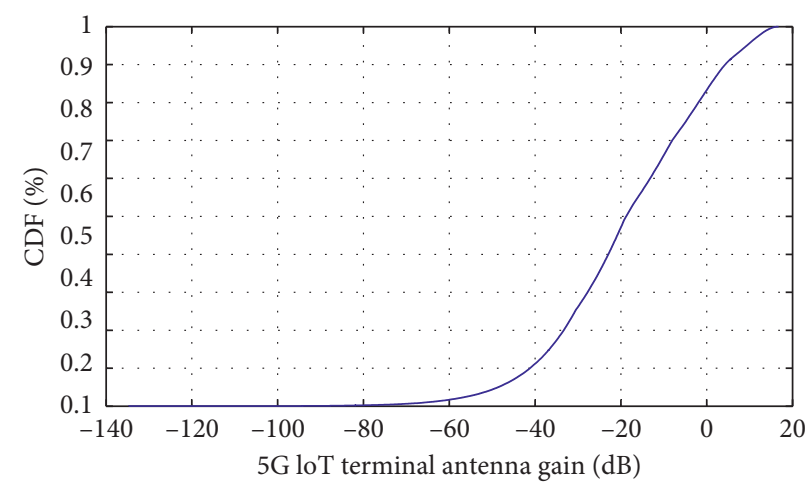

(b)

FIgURE 7: Transmitter antenna gain to the ES receiver. (a) 5G BS transmitter. (b) 5G IoT terminal transmitter.

TABLE 6: Interference margin from 5G BS (antenna pattern: $8 \times 16$ ) to FSS ES.

\begin{tabular}{lcccc}
\hline $\begin{array}{l}\text { Interference } \\
\text { criteria }\end{array}$ & Height of ES $(\mathrm{m})$ & \multicolumn{2}{c}{$\begin{array}{c}\text { D } \\
\text { isolain with } \\
\text { isolion } \\
\text { distance }(L) \mathrm{km}\end{array}$} \\
$I / N_{\mathrm{thr}}$ & $I_{\mathrm{thr}}$ & & $L=1$ & $L=0.4$ \\
\hline \multirow{2}{*}{-10} & -124.6 & 4.5 & 27.0 & 20.0 \\
& \multirow{2}{*}{-1.3} & 10 & 27.1 & 20.8 \\
\hline
\end{tabular}

TABLE 7: Interference margin from 5G BS (antenna pattern: $16 \times 16$ ) to FSS ES.

\begin{tabular}{|c|c|c|c|c|}
\hline \multicolumn{2}{|c|}{$\begin{array}{l}\text { Interference } \\
\text { criteria }\end{array}$} & \multirow[t]{2}{*}{ Height of ES (m) } & \multicolumn{2}{|c|}{$\begin{array}{c}D_{\text {margin }} \text { with } \\
\text { isolation } \\
\text { distance }(L) \mathrm{km}\end{array}$} \\
\hline$I / N_{\mathrm{thr}}$ & $I_{\text {thr }}$ & & $L=1$ & $L=0.4$ \\
\hline-10 & -124.6 & $\begin{array}{l}4.5 \\
10\end{array}$ & $\begin{array}{l}26.4 \\
26.8\end{array}$ & $\begin{array}{l}19.3 \\
21.2\end{array}$ \\
\hline-1.3 & -115.9 & $\begin{array}{l}4.5 \\
10\end{array}$ & $\begin{array}{l}31.9 \\
32.3\end{array}$ & $\begin{array}{l}26.9 \\
28.5\end{array}$ \\
\hline
\end{tabular}

TABLE 8: Interference margin from 5G IoT terminal (antenna pattern: $4 \times 4$ ) to FSS ES.

\begin{tabular}{|c|c|c|c|c|}
\hline \multicolumn{2}{|c|}{$\begin{array}{l}\text { Interference } \\
\text { criteria }(\mathrm{dB})\end{array}$} & \multirow[t]{2}{*}{ Height of ES (m) } & \multicolumn{2}{|c|}{$\begin{array}{c}D_{\text {margin }} \text { with } \\
\text { isolation } \\
\text { distance }(L) \mathrm{km}\end{array}$} \\
\hline$I / N_{\text {thr }}$ & $I_{\text {thr }}$ & & $L=1$ & $L=0.4$ \\
\hline \multirow{2}{*}{-10} & \multirow{2}{*}{-124.6} & 4.5 & 42.5 & 36.7 \\
\hline & & 10 & 42.4 & 36.9 \\
\hline \multirow{2}{*}{-1.3} & \multirow{2}{*}{-115.9} & 4.5 & 48.0 & 44.0 \\
\hline & & 10 & 47.8 & 44.1 \\
\hline
\end{tabular}

values of $I / N_{\text {thr }}$ refer to the classifications of different cases, in which the best and normal cases are simulated.

Obvious differences in the results can be found by comparing the $8 \times 16$ and $16 \times 16$ antenna patterns in $\mathrm{Ta}$ bles 6 and 7. Although the transmission power of the $16 \times 16$ antenna pattern is approximately only $1.3 \mathrm{~dB}$ less than that of the $8 \times 16$ pattern, a higher transmission power of $3 \mathrm{~dB}$ can be found in the BS configured with the $16 \times 16$ antenna pattern because the number of antennas is two times greater than the BS configured with the $8 \times 16$ antenna pattern. The trend indicates that massive MIMO technology in mmWave can be seen as a mitigation method that sharply drops the power from the interference link. More specifically, the worse the interference margin, the greater the number of antenna elements; however, the degree of the decreasing trend in the margin is slight. The main reason for this is that, in practice, both narrower beams and more complete space diversity result in the decline of interference power.

On the other hand, it is a common view that the longer the isolation distance, the better the necessary interference margin; a gain of around 4 to $7 \mathrm{~dB}$ at a $1 \mathrm{~km}$ distance can be seen by comparing the cases of $0.4 \mathrm{~km}$ isolation distance in Tables 7 and 8. Moreover, the interference margin in the normal case $\left(I / N_{\text {thr }}=-10 \mathrm{~dB}\right)$ is considerably lower than that in the best case $\left(I / N_{\text {thr }}=-1.3 \mathrm{~dB}\right)$. However, it is a significantly low probability that the best case will appear most times. Furthermore, a higher ES also slightly promotes the value of the margin, which means that improving the height is almost meaningless. The main reason for this is that the difference of varying heights of ES is not enough to effectively block the power of each interference link from the $5 \mathrm{G}$ BSs and IoT terminal, although there is an increase in the ES height of more than two times. Finally, compared with the interference links from IoT terminal to ES, the interference margins of the BSs are worse; losses of around $15.2 \mathrm{~dB}$ can be observed. 
From these three tables above, all the results of interference margin indicate that the 5G IoT system and FSS system can coexist without generating intolerance interference to the FSS system. However, more serious interference from the $5 \mathrm{G} \mathrm{BSs}$, not the IoT terminals, is also indicated in the tables due to the higher transmission power and the greater number of antennas in the sharing study between the 5G IoT system and the FSS system.

\section{Conclusions and Future Research}

This paper performed a detailed analysis of a coexistence scenario for the $5 \mathrm{G}$ IoT system in mmWave band, namely co-channel sharing with FSS downlink at $40 \mathrm{GHz}$, depending on varied separation distances, heights of ES, and interference criteria. The first part of the paper classified the interference links as BS to ES, or IoT terminal to ES, and discussed a sharing study scenario in $40 \mathrm{GHz}$. Additionally, both the 5G IoT and FSS system models and their related antenna patterns were researched. It was then demonstrated that the 5G IoT system can totally meet the interference protection criteria of the FSS system by transmitting from at least several hundred BSs and thousands of IoT terminals simultaneously. The final results also proved that the proposed massive MIMO technology can effectively suppress the interference at the ES while maintaining operable performance of the $5 \mathrm{G}$ IoT system in mmWave.

Notably, this type of coexistence analysis is not only essential but also mandatory to confirm whether the $5 \mathrm{G}$ IoT system can intelligently share spectrum with other incumbent systems, such as the FSS. This research is required for the introduction of new frequency bands during the $5 \mathrm{G}$ period and can steer how the $5 \mathrm{G}$ IoT system with mmWave can be deployed in the future.

The following points are of interest for our future research directions. First, it is a natural and significant step to conduct similar coexistence analyses for higher-potential spectrum bands, such as those above $52.6 \mathrm{GHz}$. In addition, the advanced antenna solutions and mitigation schemes are indispensable to obtain better interference margins in spectrum sharing studies. Finally, it is also important to consider a 5G IoT system coexisting with other services, such as WiFi.

\section{Data Availability}

The data used to support the findings of this study are included within the article.

\section{Conflicts of Interest}

The authors declare that they have no conflicts of interest.

\section{References}

[1] L. J. Ippolito, Satellite Communications Systems Engineering: Atmospheric Effects, Satellite Link Design and System Performance, Wiley Telecommunications, Hoboken, NJ, USA, 2017.

[2] X. Meng, J. Li, D. Zhou, and D. Yang, "5G technology requirements and related test environments for evaluation," China Communications, vol. 13, no. 2, pp. 42-51, 2016.
[3] S. Sun, M. Kadoch, L. Gong, and B. Rong, "Integrating network function virtualization with SDR and SDN for 4G/5G networks," IEEE Network, vol. 29, no. 3, pp. 54-59, 2015.

[4] M. R. Palattella, M. Dohler, A. Grieco et al., "Internet of things in the 5G era: enablers, architecture, and business models," IEEE Journal on Selected Areas in Communications, vol. 34, no. 3, pp. 510-527, 2016.

[5] Y. Wu, B. Rong, K. Salehian, and G. Gagnon, "Cloud transmission: a new spectrum-reuse friendly digital terrestrial broadcasting transmission system," IEEE Transactions on Broadcasting, vol. 58, no. 3, pp. 329-337, 2012.

[6] S. Kim and S. Kota, "ITU-R standardization of fixed satellite services (FSS)," in Proceedings of the International Workshop on Satellite \& Space Communications IEEE, pp. 247-252, Salzburg, Austria, September 2007.

[7] S. Kim, E. Visotsky, P. Moorut, K. Bechta, A. Ghosh, and C. Dietrich, "Coexistence of $5 \mathrm{G}$ with the incumbents in the 28 and $70 \mathrm{GHz}$ bands," IEEE Journal on Selected Areas in Communications, vol. 35, no. 6, pp. 1254-1268, 2017.

[8] H. Shuai, X. Sai, M. Weixiao, and C. Li, "Dense-device-enabled cooperative networks for efficient and secure transmission," IEEE Network, vol. 32, no. 2, pp. 100-106.

[9] H. Shuai, X. Sai, M. Weixiao, and C. Li, "An agile confidential transmission strategy combining big data driven cluster and OBF," IEEE Transactions on Vehicular Technology, vol. 66, no. 11 , pp. 10259-10270.

[10] W. A. Hassan, H. S. Jo, and T. A. Rahman, "The feasibility of coexistence between $5 \mathrm{G}$ and existing services in the IMT-2020 candidate bands in Malaysia," IEEE Access, vol. 5, pp. 1486714888, 2017.

[11] F. Guidolin and M. Nekovee, "Investigating spectrum sharing between 5G millimeter wave network and fixed satellite system," in Proceedings of the IEEE Globecom Workshops, pp. 1-7, San Diego, CA, USA, December 2015.

[12] H. Shuai Han, Z. Yi, M. Weixiao, and H.-H. Chen, "Selfinterference-cancelation-based SLNR precoding design for full-duplex relay-assisted system," IEEE Transactions on Vehicular Technology, vol. 67, no. 9, pp. 8249-8262, 2018.

[13] J. Kim, L. Xian, and A. S. Sadri, "Numerical simulation study for frequency sharing between micro-cellular systems and fixed service systems in millimeter-wave bands," IEEE Access, vol. 4, no. 99, pp. 9847-9859, 2016.

[14] B. Rong, Y. Qian, K. Lu, H.-H. Chen, and M. Guizani, "Call admission control optimization in WiMAX networks," IEEE Transactions on Vehicular Technology, vol. 57, no. 4, pp. 25092522, 2008.

[15] W. Weidong, Z. Fei, H. Wei, B. Wang, and Y. Zhang, "Coexistence studies between LTE system and earth station of fixed satellite service in the $3400-3600 \mathrm{MHz}$ frequency bands in China," in Proceedings of the IEEE International Conference on Broadband Network and Multimedia Technology (IC-BNMT), vol. 1125, no. 1130, pp. 26-28, Beijing, China, October 2010.

[16] W. Hong, K. H. Baek, Y. Lee, Y. Kim, and S.-T. Ko, "Study and prototyping of practically large scale mmWave antenna system for $5 \mathrm{G}$ cellular devices," IEEE Communication Magazine, vol. 52, no. 9, pp. 63-69, 2014.

[17] X. Meng, Y. Sun, D. Li et al., "Evaluated methodologies and results of IMT-2020 cellular system on millimeters waves," Journal of Beijing University of Posts and Telecommunication, vol. 41, no. 6, pp. 1-5, 2018.

[18] H. Shuai, H. Yiteng, M. Weixiao, C. Li, N. Xu, and D. Chen, "Optimal power allocation for SCMA downlink systems based on maximum capacity," IEEE Transactions on Communications, vol. 67 , no. 2, pp. 1480-1489. 


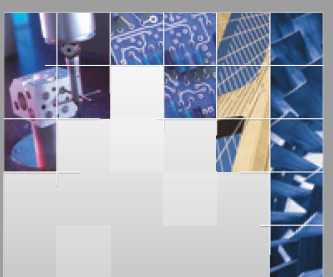

\section{Enfincering}
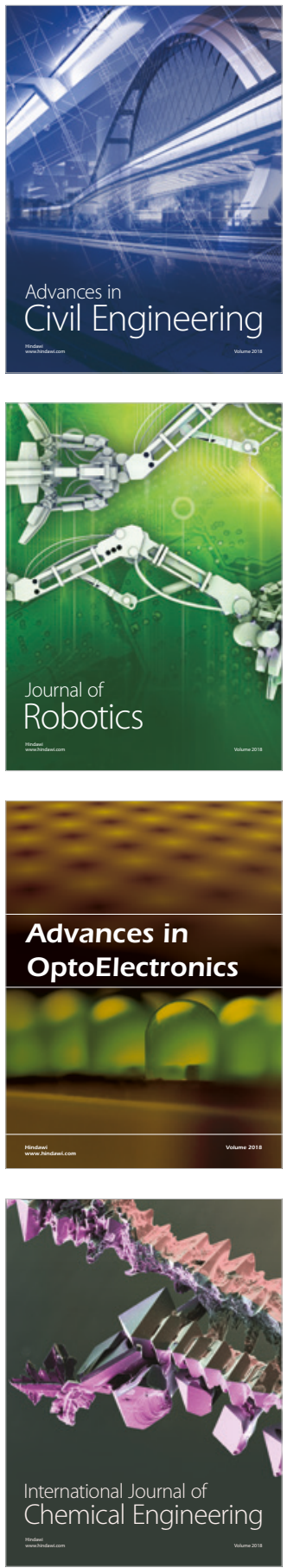

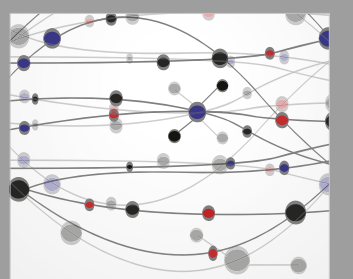

\section{Rotating \\ Machinery}

The Scientific World Journal

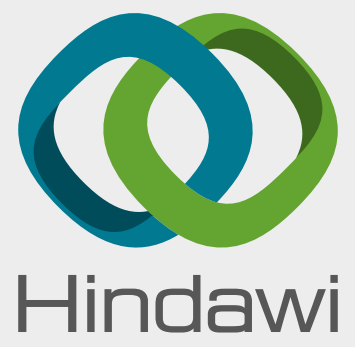

Submit your manuscripts at

www.hindawi.com
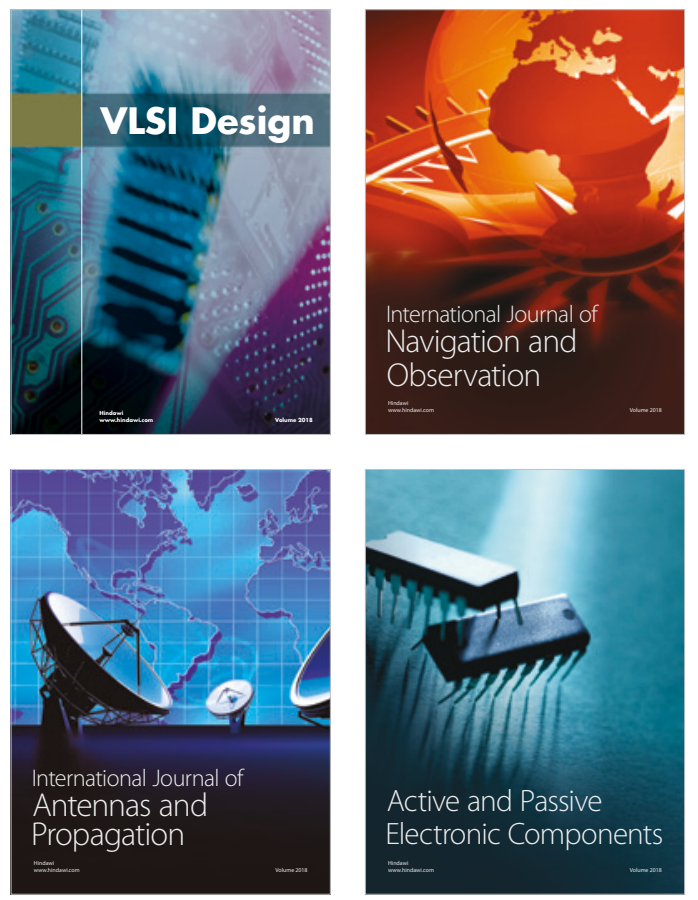
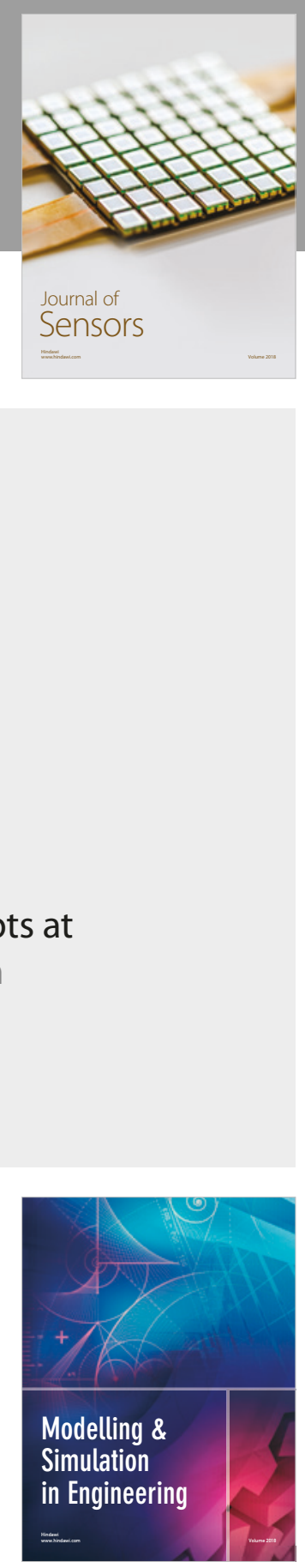

\section{Advances \\ Multimedia}
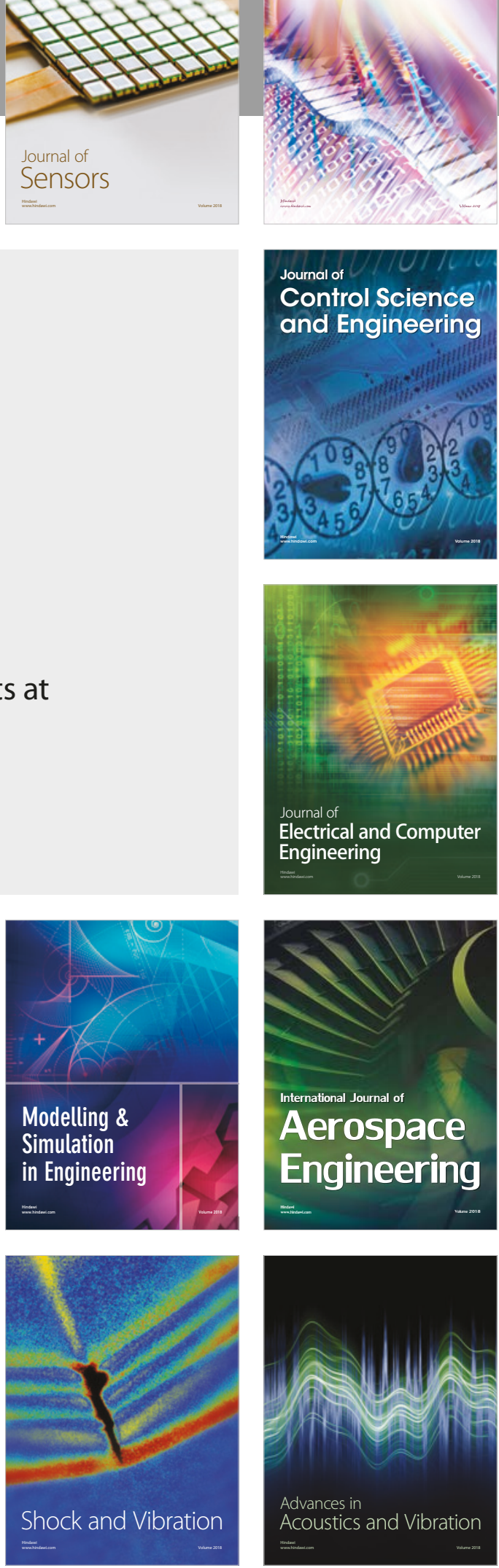